\title{
Impacts of Filter-Nonlinearities and Voltage Limitations on a Wide-Bandgap Inverter with Actively Damped LC-Filter
}

\author{
F. Maislinger ${ }^{*}$ H. Ertl ${ }^{*}$ E. Gerstbauer* \\ ${ }^{*}$ Institute of Energy Systems and Electrical Drives, TU Wien, \\ Gusshausstraße 27-29, 1040 Vienna, Austria \\ (franz.maislinger@tuwien.ac.at)
}

Keywords: Inverter drives, multi-loop-control, active damping, system input limitation, nonlinear filters.

\section{INTRODUCTION}

Today, motor inverters in the $\mathrm{kW}$ domain typically are implemented using silicon $(\mathrm{Si})$ based insulated gate bipolar transistors (Si-IGBT) operating in pulse width modulation (PWM) mode at switching frequencies up to $20 \mathrm{kHz}$. During the past few years, however, wide-bandgap switching devices like GaN- and SiC-MOSFETs have been significantly improved, especially concerning voltage capability of GaN devices. Due to the low switching- and also low onstate losses of GaN MOSFETs in comparison to Si-IGBTs, motor inverters with rather high switching frequencies but also high efficiency rates can be achieved (Shirabe and Swamy, 2012). However, the occurring high switching speed of the transistors with rise times in the range of 10 ns also create some crucial issues for motor applications caused by high $\mathrm{du} / \mathrm{dt}$ rates.

GaN Power Stage Active-Damped Filter

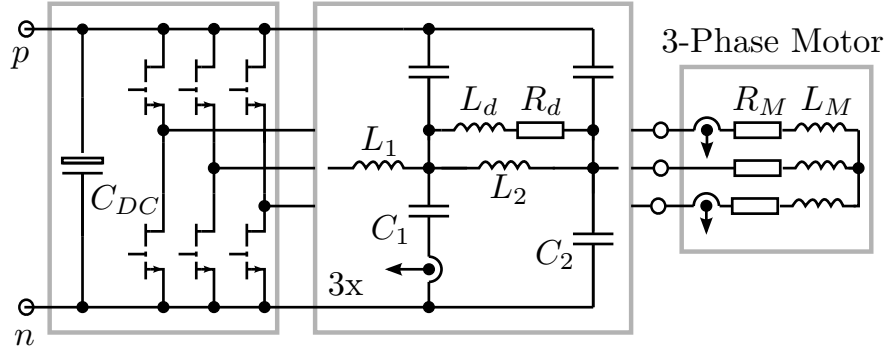

Fig. 1. Schematic concept of the proposed motor inverter with $\mathrm{GaN}$ power stage (switching frequency $100 \mathrm{kHz}$ ), active damped filter and a 3 -phase motor as load.

To avoid and reduce negative effects of high-speed switching, the GaN inverter has to be extended by a filter system, which suppresses all switching noise at the inverter's output such that motor and cabling are fed by "sinusoidallike" voltages (Fig.1). A two-stage LC output filter is used to achieve sufficient attenuation of the switching frequency harmonics. To obtain higher inverter efficiencies, an active damping concept of the LC-filter by feedback of the capacitor filter currents is applied instead of dissipative damping paths (which would result in additional losses). A closed-loop control concept using a simple PItype controller employing additional feedback of the capacitor currents is performed. The determination of fitting controller parameters will be discussed in an upcoming paper, because here, the focus lies on the active damping scheme. Therefore, a mathematical model which represents the the physical properties of the filter and the motor as a load is implemented. Nevertheless, as illustrated in the following section inverter voltage limitations and nonlinearities of passive filter elements have negative influence on the chosen active damping scheme.

\section{MATHEMATICAL MODEL}
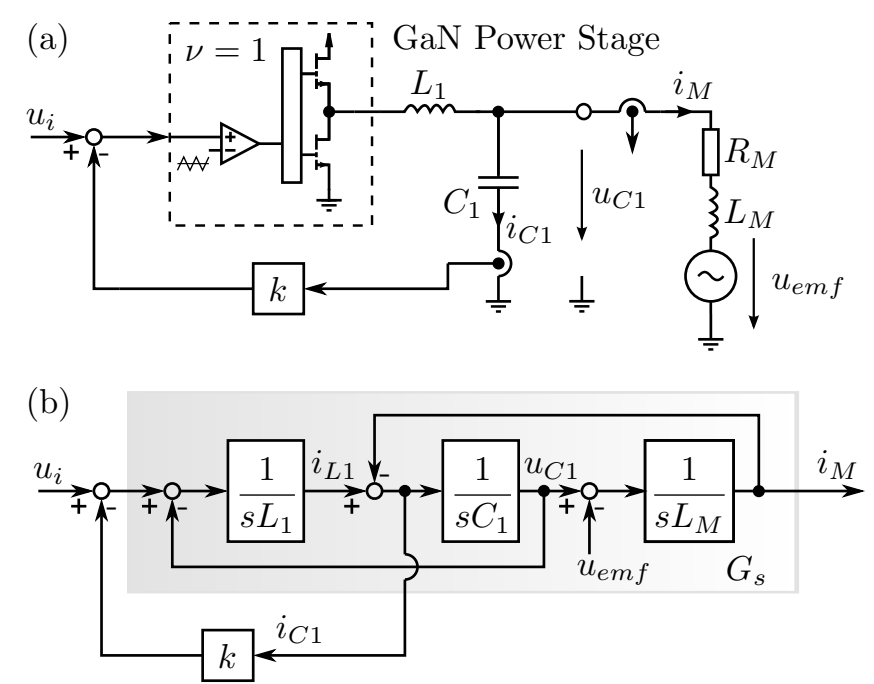

Fig. 2. (a) Single equivalent circuit of the inverter. (b) Plant dynamic model.

As described in (Maislinger et al., 2017), a single equivalent circuit can be found, (c.f., Fig. 2a), which shows the influence of the feedback of the capacitor current on the transfer function of the inverter. Therefore, the GaN power stage block has an assumed transfer function of gain $\nu=1$. Furthermore, only a single stage filter is considered. The feedback gain $k$ can be adjusted to obtain a desired filter behavior. The motor, which operates as inverter load, is considered by its resistance $R_{M}$, inductance $L_{M}$ (which is at least ten times higher than $L_{1}$ ) and by the rotational speed proportional induced voltage $u_{e m f}$, which acts in the system as a disturbance value. In case of a motor inverter 
(a)
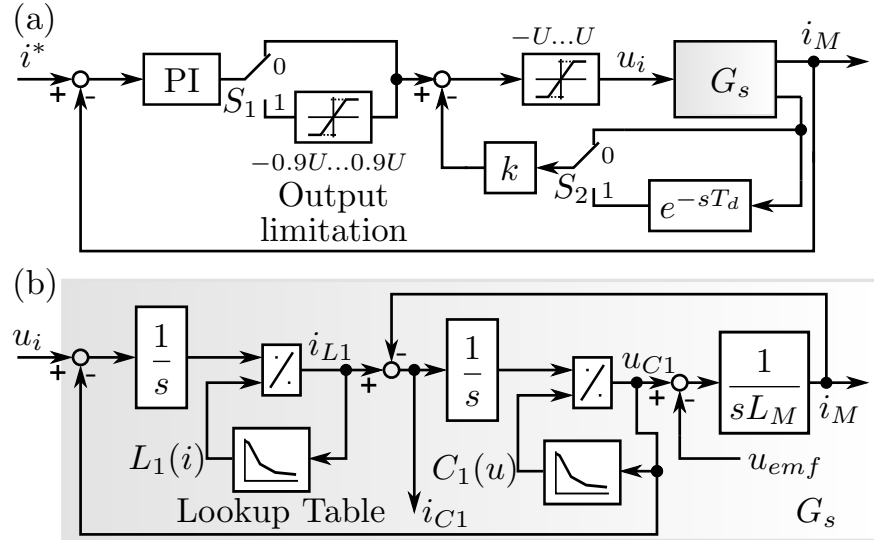

Fig. 3. (a) Closed-loop control concept. (b) Nonlinear plant dynamic model.

with constant values of $L_{1}$ and $C_{1}$, the transfer function in terms of the phase current $i_{M}$ to the converter input voltage $u_{i}$ can be written as (see Fig. $2 \mathrm{~b}$ )

$$
G(s)=\frac{1}{s\left(C_{1} L_{1} L_{M} s^{2}+C_{1} L_{M} k s+L_{1}+L_{M}\right)},
$$

where the motor resistance $R_{M}$ is set to zero (worst case scenario). A comparison of the transfer function to a conventional PT2 in the form

$$
G_{P T 2}=\frac{1}{s^{2} / \omega_{0}^{2}+2 \xi s / \omega_{0}+1},
$$

leads to an expression of the active damping parameter

$$
k=\frac{2 \xi}{\omega_{0}} \frac{L_{1}+L_{M}}{C_{1} L_{M}}=2 \xi \sqrt{\frac{\left(L_{1}+L_{M}\right) L_{1}}{C_{1} L_{M}}},
$$

which guarantees a well damped system behavior.

\subsection{System Input Limitation}

For the investigated inverter, in case of a large stepchange in the desired motor current $i^{*}$ the system input $u_{i}$ is limited to $\pm U$ (whereby $U$ corresponds to the half of the DC-link voltage) as illustrated in Fig. 3a (switch $S_{1}$ is in position $S_{1}=0$ ). Therefore, the implemented active damping scheme is ineffective, since no control margin is available to counteract the excitation of the filter resonance. This phenomenon can be decreased if an additional output limiter is adapted next to the controller $\left(S_{1}=1\right)$, which guarantees a buffer for the active damping part. Fig. 4a depicts the influence of the system input limitation on the filter current through the inductor $i_{L 1}$ as well as the resulting motor current, for both cases. As can be seen, the additional output limiter decreases the current in the passive filter elements, but also reduces the system dynamic.

\subsection{Nonlinearities of Passive Filter Elements}

To consider nonlinearities of the passive filter elements in the model, the function $G_{s}$ of Fig. $2 \mathrm{~b}$ has to be replaced by Fig. 3b. The values of the lookup tables come from data sheets that correspond to a Sendust powder core with a permeability of 60 for $L_{1}(i)$ and an Arcshield ceramic capacitor with $330 \mathrm{nF}, 500 \mathrm{~V}$ for $C_{1}(u)$ (KEMET, 2017). As mentioned above in (3), the optimal active damping parameter $k$ is a function of the passive filter elements $L_{1}$ and $C_{1}$. However, by measuring the capacitor filter current $i_{C 1}$ nonlinearities of $L_{1}$ and $C_{1}$ seem to have only small influences on the active damping mechanism of the proposed inverter as illustrated in Fig. 4b. But, if the effect of a time delay caused by measuring the filter current is also considered $\left(S_{2}=1\right)$, the impact of nonlinear filter elements is crucial. In Fig. $4 \mathrm{~b}$, a time delay $T_{d}$ of $5 \mathrm{us}$ is assumed, which corresponds to half a period at a switching frequency of $100 \mathrm{kHz}$. For higher time delays in the range of a full switching period the system becomes unstable.
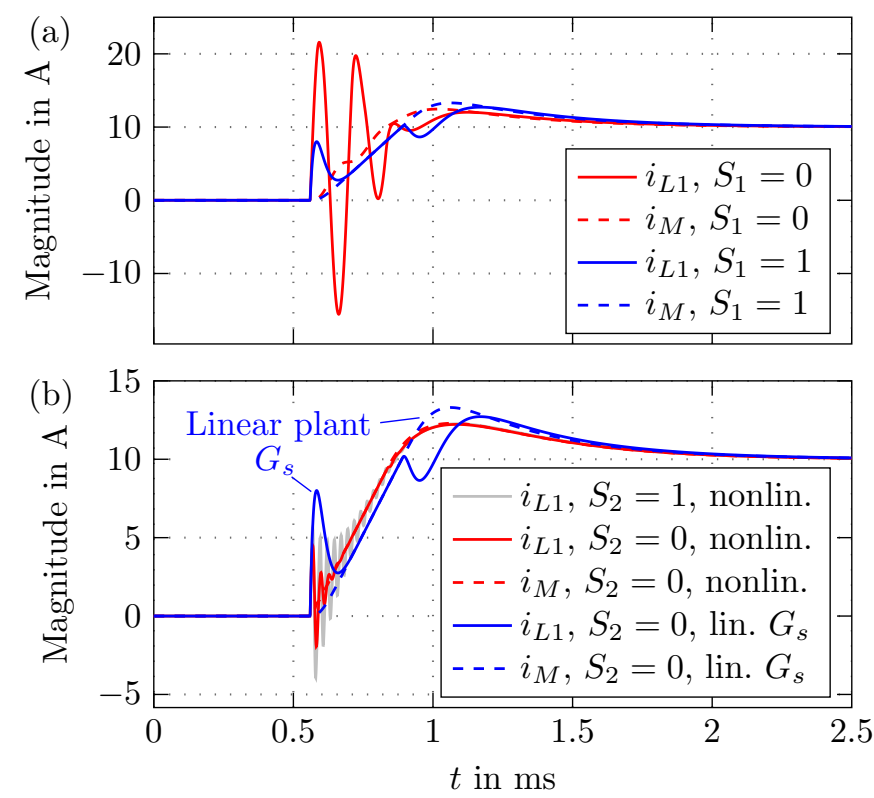

Fig. 4. (a) Influence of system input limitation. (b) Impact of nonlinearities on filter- and motor currents (red) as well as influence of time delay in capacitor current measurement (gray). In all cases: $S_{1}=1$.

\section{CONCLUSION}

The paper gives a brief overview about the impacts of nonlinearities and input limitations on wide-bandgap inverters with an active damped LC-filter. It is shown that by using a further limiter after the controller output, the functionality of the active damping scheme remains present, independent of the controller output. Furthermore, nonlinearities and time delays in capacitor current measurement can lead to an unstable behavior of the dynamic plant. The negative time delay effect can be neglected, if a linear observerbased model is used to calculate the capacitor filter current instead of the measurement. The effect of nonlinear filter elements on the control concept with the observer-based model is under investigation.

\section{REFERENCES}

KEMET (2017). K-sim version 2.0.17.8. Internet, 2017. Ksim.kemet.com.

Maislinger, F., Ertl, H., Stojcic, G., and Holzner, F. (2017). Control loop design for closed-loop class-d amplifiers with 4th order output filter. PCIM Europe 2017.

Shirabe, K. and Swamy, M. (2012). Advantages of high frequency pwm in ac motor drive applications. Energy Conversion Congress and Exposition (ECCE). 